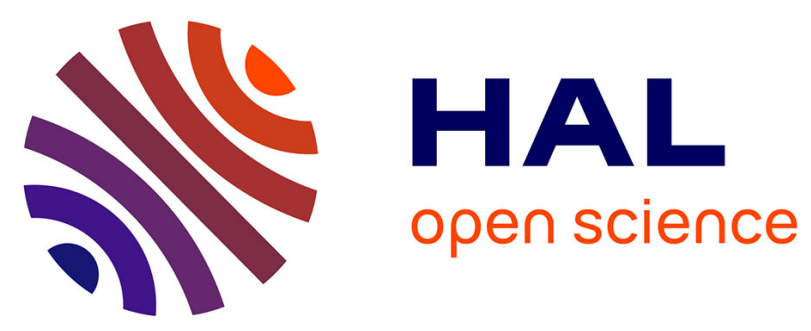

\title{
Comparison of optimization-based strategies for constrained control of Auto-Steering systems
}

Iris Ballesteros-Tolosana, Sorin Olaru, Pedro Rodriguez-Ayerbe, Guillermo Pita-Gil, Renaud Deborne

\section{- To cite this version:}

Iris Ballesteros-Tolosana, Sorin Olaru, Pedro Rodriguez-Ayerbe, Guillermo Pita-Gil, Renaud Deborne. Comparison of optimization-based strategies for constrained control of Auto-Steering systems. 15th European Control Conference (ECC 2016), Jun 2016, Aalborg, Denmark. 10.1109/ecc.2016.7810518 . hal-01429244

HAL Id: hal-01429244

https://hal-centralesupelec.archives-ouvertes.fr/hal-01429244

Submitted on 7 Jan 2017

HAL is a multi-disciplinary open access archive for the deposit and dissemination of scientific research documents, whether they are published or not. The documents may come from teaching and research institutions in France or abroad, or from public or private research centers.
L'archive ouverte pluridisciplinaire HAL, est destinée au dépôt et à la diffusion de documents scientifiques de niveau recherche, publiés ou non, émanant des établissements d'enseignement et de recherche français ou étrangers, des laboratoires publics ou privés. 


\title{
Comparison of optimization-based strategies for constrained control of Auto-Steering systems
}

\author{
Iris Ballesteros-Tolosana ${ }^{1,2}$ Sorin Olaru ${ }^{1}$, Pedro Rodriguez-Ayerbe ${ }^{1}$, Guillermo Pita-Gil ${ }^{2}$, Renaud Deborne ${ }^{2}$
}

\begin{abstract}
This paper presents a comparison between two approaches addressing vehicle lateral dynamics control. In the framework of Auto-Steering for target tracking application, Model Predictive Control (MPC) and Interpolation Based Control (IBC) are studied on a similar model-based design. Both control strategies are of interest by their ability to treat problem constraints and actuator limits in a systematic manner, as well as their capability to include changes of the behavior when the speed of the vehicle changes, yielding a linear parameter varying system. Speed variation for the MPC controller brings an uncertain model, that will be described by a polytopic class of dynamics. Thanks to the online measurement of the parameter, the system dynamics will be computed at each sample time to solve the optimization problem. Parameterdependent Lyapunov functions and positive set invariance theory are used to ensure stability and feasibility. Interpolation Based control is a different approach, whose principle is to use a control action constructed as an interpolation between two computed extreme values. Each time, two linear programming problems are solved, which makes this approach to be a suitable trade-off between performance and computation cost.
\end{abstract}

\section{INTRODUCTION}

In the recent years, Advanced Driving Assistance Systems (ADAS) have gained great importance in the automotive industry. These are complex control systems that are able to compensate or take control over the vehicle dynamics, in order to avoid potential dangerous situations, or spare the driver from tedious quotidian driving situations. Driving conditions are never the same, so it becomes a critical feature to come up with robust control strategies that always keep a correct performance and ensure the safety of the system by constraints handling. This study presents two different generic approaches for the control design of a vehicle AutoSteering system, where the uncertainty produced by the variation on the speed is considered explicitly.

The exposition is organized as follows. Section II provides an overview of the auto-steering system and the dynamical model used. In the subsequent, the two studied methods are detailed: in Section III Model Predictive Control is considered and continuing with the study, Interpolation Based Control is applied in Section IV. After that, Section V shows simulations that have been carried out in order to test the methods and draw the conclusions in Section VI.

\footnotetext{
1 Laboratory of Signals and Systems (L2S), Centrale-Supèlec, CNRS, Université de Paris-Saclay, France. \{sorin.olaru, pedro.rodriguez @ centralesupelec. fr

${ }^{2}$ RENAULT SAS, DEA-SAD5, Development ADAS control laws. \{iris.ballesteros-tolosana, renaud.deborne, guillermo.pita-gil\}@renault.com
}

\section{Auto-SteEring System AND CONTROL PROBlem FORMULATION}

\section{A. Auto-steering System}

Several auto-steering control systems have been developed in the literature and industry. These active control systems act on the lateral vehicle dynamics control in order to offer safety, like the Lane Keeping Assistance [1] or Stability Control Systems [2], and comfort, such as Target Tracking or Lane Centering Assistance. All of them cooperate with the longitudinal vehicle dynamics control, that is an independent control system which will be in charge of the vehicle's longitudinal speed, acceleration and distance with the preceding vehicle, if any. These longitudinal control variables are measured or estimated at each sample time and will be used as external parameters in the lateral control systems. This means that in the present work the control structure is divided in two parts, where lateral and longitudinal dynamics of the vehicle are assumed to be decoupled.

The auto-steer system object of study in this application case is comprised in the Traffic Jam Pilot (TJP), that is in charge of the vehicle steering angle at low speed when a target vehicle is detected, that is, the vehicle which runs ahead of our vehicle. The controller goal is to follow this target lateral position and yaw angle.

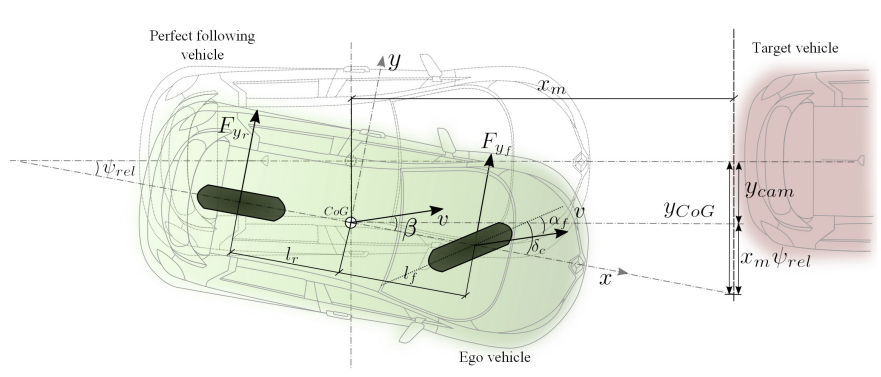

Fig. 1: Auto-steering system for target tracking

\section{B. Linear parameter varying model for the controller design}

Vehicle dynamics have been studied and targeted in different contexts, [3], [4], [5], being common knowledge in both mechanical and control fields. In [6] the commonly used models for vehicle steering control are outlined. In the present work, a dynamic model based on the interacting lateral forces between the vehicle and the road surface through the tires is used. The tire forces modeling is an extensive topic itself [7]. In this paper, linear and uncoupled tire forces 
behavior is considered with the objective of control design for normal driving conditions. We are not interested in the control of the vehicle in large slip angles and other such extreme conditions [8].

The adopted dynamical model is based on the known bicycle model, as it exhibits a good compromise between complexity and performance. In [9] the lateral dynamics of a vehicle bicycle model moving along an infinite surface are given. However, in order to represent the dynamics of our system, it is convenient to locate the model in a different reference, that is, the target vehicle and a virtual perfect following vehicle (Fig.1). Thus $\psi$ will be related to the relative angle between the perfect following vehicle and the controlled one. Following this last remark we adopt $\psi_{\text {rel }}$ for this angle and denote by $y_{C o G}$ the lateral difference between the trajectory of the ego vehicle and the perfect following one. $y_{\text {cam }}$ stands for the lateral offset between our vehicle and the target vehicle. Thereby, the tracking objective of the auto-steering control problem can then be expressed as a problem of stabilizing the dynamics of the controlled vehicle with respect to the virtual perfect following one.

After taking this into consideration, the conceived model is expressed as $\dot{x}(t)=A x(t)+B u(t), y(t)=C x(t)$ with:

$$
\begin{gathered}
A=\left(\begin{array}{cccc}
\frac{-\left(C_{f} l_{f}^{2}+C_{r} l_{r}^{2}\right)}{I_{z} v} & \frac{\left(C_{f} l_{f}-C_{r} l_{r}\right)}{I_{z}} & \frac{-\left(C_{f} l_{f}-C_{r} l_{r}\right)}{I_{z} v} & 0 \\
1 & 0 & 0 & 0 \\
\frac{\left(C_{r} l_{r}-C_{f} l_{f}\right)}{m v} & \frac{\left(C_{f}+C_{r}\right)}{m} & \frac{-\left(C_{f}+C_{r}\right)}{m v} & 0 \\
0 & 0 & 1 & 0
\end{array}\right) \\
B=\left(\begin{array}{c}
\frac{C_{f} l_{f}}{I_{z}} \\
0 \\
\frac{C_{f}}{m} \\
0
\end{array}\right) \quad C=\left(\begin{array}{cccc}
1 & 0 & 0 & 0 \\
0 & x_{m} & 0 & 1
\end{array}\right)
\end{gathered}
$$

Where $x(t)$ is the state vector $\left[\dot{\psi}, \psi_{\text {rel }}, \dot{y}_{C o G}, y_{C o G}\right]^{T} \in \mathbb{R}^{n}$, $u$ is the control input $\in \mathbb{R}^{p}$ which represents the steering wheel angle $\delta_{c}$ and $y \in \mathbb{R}^{m}$ holds by the vector of system outputs, that will be $\left[\dot{\psi}, y_{\text {cam }}\right]^{T}$. Complete measurements of the states are considered in the present study. The transition matrix $A \in \mathbb{R}^{n \times n}$ and input matrix $B \in \mathbb{R}^{n \times p}$ depend on several vehicle parameters: $C_{f}$ and $C_{r}$ denote the cornering stiffness of the front and rear wheels respectively. The distances from the front and rear axis to the center of gravity are $l_{f}$ and $l_{r}, m$ stands for the vehicle mass and $I_{z}$ for the total yaw moment of inertia. In addition to this, the observation matrix $C \in \mathbb{R}^{m \times n}$ depends on $x_{m}$ which is the longitudinal distance with respect to the followed target. All of them are considered known and fixed, so they do not introduce any uncertainty to the model.

Finally, one of the main parameters appearing in (1) is the speed of the vehicle. In practice, the varying parameter will be defined as the inverse of the longitudinal speed of the vehicle, $\nu=1 / v$. The speed range in which the auto-steer system will be active, [1-40 km/h], is known, so the parameter is bounded, $\nu \in \mathbb{V}$. Its time-varying (and measured) characteristic yields a linear parameter varying
(LPV) model. In the following, discrete-time formulation is used. For doing so, the forward Euler discretization method based on a truncated Taylor series expansion with $T s=$ $0.01 \mathrm{~s}$ has been applied to the continuous-time system (1).

There are several uncertainty sources when modeling a system, and consequently various ways to describe them when considered in the control problem formulation [10], [11]. In this paper, a linear parameter-varying formulation will be considered which can in turn be embedded in a polytopic uncertainty approach, as any operating speed can be obtained as a convex combination of the maximal and minimal ones due to the monotonicity of the curve. For prediction purposes, the system dynamics will be described by the parameter varying model

$$
\begin{aligned}
x_{k+1} & =A\left(\nu_{k}\right) x_{k}+B u_{k} \\
y_{k} & =C x_{k}
\end{aligned}
$$

With $A\left(\nu_{k}\right), B, C$ described by the discrete time formulation of (1), and $A\left(\nu_{k}\right)=\sum_{i=1}^{n_{v}} \alpha_{i} A\left(\nu_{i}\right)$ with $\sum_{i=1}^{n_{v}} \alpha_{i}=1, \alpha_{i} \geq$ 1 and $n_{v}=2$.

\section{Constrained control objectives}

Industrial applications involve constraints that need to be taken into account at the design stage in order to fulfill security conditions or actuator limitations. The control design methods considered next are appealing for this constraint handling capability, which allows the system to guarantee a correct performance and to work closer to the limits without running any risks. In practice the dynamical system has limitations with respect to the states, outputs and in the control action, that is

$$
x \in X, y \in Y, u \in U
$$

where $X \subset \mathbb{R}^{n}, Y \subset \mathbb{R}^{m}$ and $U \subset \mathbb{R}^{p}$ are the bounded sets of admissible states, outputs and inputs, respectively, all of them containing the origin in their interior..

\section{Constrained Model Predictive Control}

\section{A. Problem formulation}

A conventional Model Predictive Control (MPC) strategy is used [12]. Each time, a finite horizon Optimal Control Problem (OCP) is formulated, which in this case consists of solving a quadratic programming (QP) to minimize the control input rate along the prediction horizon subject to linear equality and inequality constraints.

$$
\begin{aligned}
\min _{\Delta U} J\left(\bar{x}_{k}, \Delta U\right) & =\left\|\bar{x}_{N}\right\|_{P}^{2}+\sum_{k=1}^{N-1}\left\|\bar{x}_{k}\right\|_{Q}^{2}+\left\|\Delta u_{k}\right\|_{R}^{2} \\
\text { s.t. } & \bar{x}_{k+1}=\bar{A}\left(\nu_{k}\right) \bar{x}_{k}+\bar{B} \Delta u_{k} \\
& y_{k}=\bar{C} \bar{x}_{k} \\
& \bar{x} \in \bar{X}, y \in Y, \Delta u \in \Delta U \\
& \bar{x}_{N} \in \Omega
\end{aligned}
$$

where $\Delta U=\left[\Delta u_{k}^{T}, \Delta u_{k+1}^{T}, \ldots, \Delta u_{k+N-1}^{T}\right]^{T}$, using an augmented state formulation, with $\bar{x}_{k}^{T}=\left[\begin{array}{ll}x_{k}^{T} & u_{k-1}^{T}\end{array}\right]^{T}$ and considering the known velocity form, $\Delta u_{k}=u_{k}-u_{k-1}$, 
which has additional interesting properties from the offset free tracking point of view [13]. $Q \succ 0$ and $R \succ 0$ represent respectively the positive definite state and input weighting matrices along the prediction horizon $N$ [14]. Moreover, a quadratic terminal cost and terminal set $\Omega$ associated to an stabilizing gain for the unconstrained system are considered to ensure system's recursive stability and admissibility with respect to constraints and will be further studied in (III-B). Finally, in order to formulate this finite-time OCP, only the current speed is known (II-B), so the system matrices in (2) are computed at each sample time and are considered constant along the prediction horizon based on the measured $\nu_{k}$. This constant parameter profile along the prediction horizon is feasible with respect to the available information and preferable to a robust MPC design which is conservative from the performance point of view.

\section{B. Feasibility, cost function and stability}

A classical construction for the guarantee of stability and recursive feasibility in predictive control is the use of the so-called terminal set - terminal cost in the design [15]. These constraints are intended to transform the cost function into a pseudo-infinite cost-to-go and thus play the role of a Lyapunov function for the closed loop system.

1) Terminal cost and stabilizing gain for the unconstrained system: Taking into account the parameter-varying nature of the dynamics, the terminal cost will be based on the construction of a parameter-dependent Lyapunov function. In [16] necessary and sufficient conditions for the computation of such functions are derived. Then, in [17] this formulation is modified in order to achieve a finite-time receding-horizon formulation, by means of enforcing a cost decrease leading to a parameter-varying terminal cost and a stabilizing control law for the unconstrained system. The LMI designed to obtain a parameter-dependent terminal cost for $\nu \in \mathbb{V}$ is recalled here for completeness. We point the interested readers to [17] - [18] for further discussions on the subject and proof for Lemma 1.

Lemma 1: Let the symmetric positive definite matrices $S_{i}$, $S_{l}$ and $G_{i}, Y_{i}, Y_{l}$ of appropriate dimensions, be such that

$$
\left[\begin{array}{cccc}
G_{i}+G_{i}{ }^{T}-S_{i} & \left(A_{i} G_{i}+B Y_{i}\right)^{T} & Y_{i}^{T} & G_{i}{ }^{T} \\
\left(A_{i} G_{i}+B Y_{i}\right) & S_{l} & 0 & 0 \\
Y_{i} & 0 & R^{-1} & 0 \\
G_{i} & 0 & 0 & Q^{-1}
\end{array}\right]>0
$$

for all $i=1, \ldots, n_{v}$ and $l=1, \ldots, n_{v}$. Then $G_{i}$ is full rank and the parameter-dependent Lyapunov function is given by $P_{i}=S_{i}^{-1}$, and the parameter-dependent stabilizing gain by $K_{i}=Y_{i} G_{i}^{-1}$ which yields the stabilizing parameter dependent control law $u_{k}=\sum_{i=1}^{n_{v}} K_{i}(\nu) x_{k}$.

The number of degrees of freedom introduced in the problem through the matrices $G_{i}, S_{i}, S_{l}$ can be adapted to obtain a common Lyapunov function for all the parameter values, denoted $\bar{P}$, by imposing $S_{i}=S_{l}=S$ and $G_{i}=G$ or a parameter-dependent one $P(\nu)$, that will change depending on the current measured value of the speed.
2) Invariant sets: Set invariance theory is largely used in control applications [19], in order to assess critical features such as feasibility, stability or robustness.

Maximal output admissible set (MOAS) [20]: considering a constrained stable linear dynamical system $x_{k+1}=$ $A_{c l} x_{k}, y_{k}=C x_{k}$, a set $\Omega$ is output admissible if $x_{0} \in \Omega$ implies that $x_{k} \in \Omega$ for all future times, $k>0$ and no constraints violation takes place. This is equivalent to $\Omega=\left\{x \in \mathbb{R}^{n}: C A_{c l}^{k} x \in Y\right\}$. We are interested in transition matrices $A_{c l}$, corresponding to the closed loop form, that is, $\bar{A}(\nu)+B K(\nu)$ where the stabilization is done with the parameter-dependent gain $K(\nu)$ (Lemma.1). In [20] and [21], the properties of this kind of robust invariant sets are characterized and recursive algorithms are introduced in order to compute them.

Two MOAS have been computed, $\Omega_{\bar{P}}=\left\{x \in \mathbb{R}^{n}\right.$ : $\left.H_{\Omega_{\bar{P}}} x \leq b_{\Omega_{\bar{P}}}\right\}$ and $\Omega_{\bar{P}(\nu)}\left\{x \in \mathbb{R}^{n}: H_{\Omega_{\bar{P}(\nu)}} x \leq b_{\Omega_{\bar{P}(\nu)}}\right\}$. Both are tested in the present study as terminal sets for the optimization problem (4). For $\Omega_{\bar{P}}$, the parameter-dependent stabilizing gain is obtained with the common Lyapunov function $\bar{P} . \Omega_{\bar{P}(\nu)}$ is computed using a parameter-varying control based on the parameter-varying Lyapunov function $\bar{P}(\nu)$. As expected, $\Omega_{\bar{P}}$ proves to be quite restrictive $\left(\Omega_{\bar{P}} \subset\right.$ $\left.\Omega_{\bar{P}(\nu)}\right)$. This is further analyzed in Section (V).

N-step controlled invariant set (CIS): given a positive invariant set $\Omega \subset X$ with respect to a pre-defined control law, a set $\mathcal{C}_{N}$ is $\mathrm{N}$-step controlled invariant if any $x_{0} \in \mathcal{C}_{N}$ can be driven to $\Omega$ in $\mathrm{N}$-steps while satisfying the constraints by applying admissible control inputs, $u_{k} \in U$. This set can be computed for the constrained LPV system (2), (3), by means of recursive pre-image set construction with respect to $\Omega$ and for each extreme realization of the polytopic uncertainty description [22], [23]. Such a N-step controlled set is denoted here by $\mathcal{C}_{N}=\left\{x \in \mathbb{R}^{n}: H_{C} x \leq g_{C}\right\}$.

If the procedure is initiated with a robust control invariant set $\Omega$ then $\mathcal{C}_{N}$ is robust control invariant. Further details on such constructions and the available tools can be found in [21], [24].

In the case of lateral vehicle dynamics, the CIS characterization leads to a complex set in $\mathbb{R}^{5}$, and the computational load becomes excessive. Due to this limitations, the 3-steps controlled invariant set, $\mathcal{C}_{3}$ will be used in the present study. In (Fig. 2, the MOAS $\Omega_{\bar{P}(\nu)}$ and the CIS $\mathcal{C}_{3}$ for the LPV system (2) is shown via cutting with the state constraints polyhedron $X$ (3). It can be seen that the limits for $\psi_{\text {rel }}$ are relatively small compared to the admissible values at the cutting values, so the initial conditions for this state will highly condition the feasibility of the control problem. The limits for the rest of the states are close to the state constraints and are not showed here due to limited space.

3) Recursive feasibility: As mentioned above, the parameter $\nu(k)$ is known at each moment in time but no other information is available for the prediction, so the MPC problem is solved for the system related to the current speed, and considered constant along the whole prediction horizon. This is an issue with respect to the recursive feasibility, which has to consider the worst case parameter variation. 


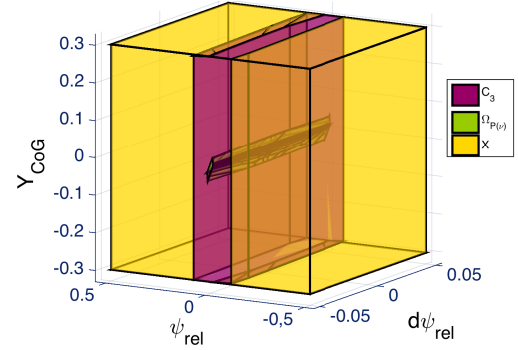

Fig. 2: Invariant sets: cut $\left[x_{2}, x_{3}, x_{4}, 0,0\right]$

Indeed, the tail of the optimal sequence would continue being feasible only if the speed is constant. In order to ensure recursive feasibility, one solution would be to take into account all the possible combinations of parameters realization along the prediction horizon. Nevertheless, this option would lead to a high computational load, as the number of possible prediction model combinations would increase exponentially with the prediction horizon length. In the present study we adopt a slightly different approach but that will not increase the computational burden. An additional constraint is considered in the nominal MPC problem (4): the one step-ahead predicted state belongs to the robust controlled invariant set (III-B.2) $x_{k+1} \in \mathcal{C}_{N}$. This ensures that independently of the parameter evaluation on the next time step, the problem remains feasible. More than that, the fact that the predicted nominal trajectory enters in the terminal set, ensures the optimality on a pseudo-infinite horizon for the time-invariant prediction model.

\section{INTERPOLATION BASED CONTROL}

This section is introduces the main idea of a relatively novel constrained control approach [25], whose online computational load is much lower than in the previous method. The main idea is to apply a control action that is interpolated between the stabilizing unconstrained controller and the control in the controlled invariant set.

\section{A. Problem formulation}

The IBC technique is based on the fact that any state inside the CIS can be expressed as the linear combination of two states: $x_{k}=\eta_{k} x_{c_{k}}+\left(1-\eta_{k}\right) x_{o_{k}}$, where $x_{c_{k}} \in \mathcal{C}_{N}, x_{o_{k}} \in \Omega$ and $0 \leq \eta \leq 1$.

In a similar way, the following control law is considered: $u_{k}=\eta_{k} u_{c_{k}}+\left(1-\eta_{k}\right) u_{o_{k}}$ where $u_{c_{k}}$ is obtained by solving an (optimization-based) control problem which exploits the controlled-invariance and $u_{o_{k}}$ will be obtained using a parameter-varying stabilizing feedback gain $K\left(\nu_{k}\right)$.

In order to obtain the input signal $u_{k}$, the first step is to compute the coefficient $\eta_{k}$. In order to do so, a nonlinear optimization problem is build, whose objective will be to minimize this coefficient in order to be as close as possible to the unconstrained local controller.

$$
\eta_{k}^{*}=\min _{x_{c_{k}}, x_{o_{k}}, \eta_{k}}\left\{\eta_{k}\right\} \quad \text { s.t. }\left\{\begin{array}{l}
H_{C} x_{c_{k}} \leq b_{C} \\
H_{\Omega} x_{o_{k}} \leq b_{\Omega} \\
x_{k}=\eta_{k} x_{c_{k}}+\left(1-\eta_{k}\right) x_{o_{k}} \\
0 \leq \eta_{k} \leq 1
\end{array}\right.
$$

To transform (6) in a LP, the following change of variables can be done [26]: $r_{c_{k}}=\eta_{k} x_{c_{k}}, r_{o_{k}}=\left(1-\eta_{k}\right) x_{o_{k}}$. Hence,

$$
\eta_{k}^{*}=\min _{r_{c_{k}}, \eta_{k}}\left\{\eta_{k}\right\} \quad \text { s.t. }\left\{\begin{array}{l}
H_{C} r_{c_{k}} \leq \eta_{k} b_{C} \\
H_{\Omega}\left(x_{k}-r_{c_{k}}\right) \leq\left(1-\eta_{k}\right) b_{\Omega} \\
0 \leq \eta_{k} \leq 1
\end{array}\right.
$$

Once the optimal interpolation factor $\eta_{k}^{*}$ is known, the states $x_{c_{k}}$ and $x_{o_{k}}$ are also obtained as a by-product. The only missing element is the admissible input signal corresponding to $x_{c_{k}}$, that is, an input signal $u_{c_{k}}$ that will keep a state that belongs to the border of $\mathcal{C}_{\mathcal{N}}$ inside it. A simple one-step optimization problem constructs this control action by optimizing the contraction factor $\gamma_{k}$ :

$$
\gamma_{k}^{*}=\min _{u_{c_{k}}, \gamma_{k}}\left\{\gamma_{k}\right\} \quad \text { s.t. }\left\{\begin{array}{l}
H_{C}\left(\bar{A}_{i} x_{c_{k}}+B u_{c_{k}}\right) \leq \gamma_{k} b_{C} \\
H_{U} u_{c_{k}} \leq b_{U} \\
0 \leq \gamma_{k}
\end{array}\right.
$$

with $i=1,2, \ldots n_{v}$. As it can be seen, the IBC method consists of a pair of linear programming problems with $n+1$ arguments, which are considerably simpler than the $N$-step MPC optimization (QP).

\section{B. Stability and feasibility}

The interpolation based control scheme ensures asymptotic stability for all initial states inside the controlled invariant set, $\mathcal{C}_{N}$, with the interpolation factor $\eta_{k}$ playing the role of a Lyapunov function on $\mathcal{C}_{N} \backslash \Omega$. By exploiting the controlledinvariance properties of $C_{N}$ it can be shown that the problem (8) is always feasible, [26].

From the construction of the invariant set in (Fig.2), the strict inclusion of the CIS, $\mathcal{C}_{N}$, in the state constraints was obvious and it could happen that the states to control lay out of its boundaries, which would bring up an unfeasible problem when solving (7) in $X \backslash \mathcal{C}_{N}$. A simple way to avoid the unfeasibility for the states out of $\mathcal{C}_{N}$, is to soften the constraints in order to contain the current state. The minimum scaled polyhedron $\mathcal{C}^{\prime}{ }_{N}$ that contains the current system state $x_{k}$ is computed form the original $\mathcal{C}_{N}$ solving the following LP:

$$
\beta_{k}^{*}=\min \{\beta\} \quad \text { s.t. } H_{C} x_{k} \leq \beta b_{C}
$$

Once $\beta_{k}^{*}$ is known, the scaled set for the current iteration is defined $\mathcal{C}^{\prime}{ }_{N}=\left\{x \in \mathbb{R}^{n}: H_{C^{\prime}} x \leq b_{C^{\prime}}\right\}$ with $H_{C^{\prime}}=H_{C}$ and $b_{C^{\prime}}=\beta_{k}^{*} b_{C}$. This artifact ensures the feasibility in (8) as long as the state constraints are feasible. However the stability will be guaranteed only for $\beta^{*}<1$. 


\section{Simulation STUdies AND ANALYSis}

In this section the two controllers derived in Sections III and IV have been tested on a extensive range of initial conditions. In order to present a relevant comparison for a usual configuration, a scenario with a trapezoidal profile of varying speed between $[1-40 \mathrm{~km} / \mathrm{h}]$ has been defined. The simulated vehicle will be running in a straight road while the ego vehicle is initialized at a perturbed initial state.

\section{A. Basic analysis of the MPC feasibility}

The safe behavior of the controller is a critical feature when driving an application in which human life is involved. We want to ensure robust feasibility of the problem from the design stage. For doing so, we have to be able to steer the system state to the terminal set $\Omega$ in $N$ steps ahead in time, fulfilling all the system input and output constraints no matter the (bounded) speed of the vehicle. This feature is influenced by three arguments of the MPC design: first, the initial conditions $x_{0}$. Second, the length of the prediction horizon $N$ and last, the size of the MOAS, $\Omega$. If we start at initial conditions that are far away from the MOAS, we will need to increase the prediction horizon, in order to get a feasible problem with $x_{N} \in \Omega$. At the same time, the size of the MOAS will make this task easier to achieve at the price of an increase complexity of the respective polyhedral set. This first test analyzes the influence of the prediction horizon length and the terminal set constraint size. For doing that, we have run a simulation starting from the possible maximum values of the initial conditions $x_{0}=[0.05,0.05,0.2,0.3,0.15]^{T}$, checking which would be the necessary prediction horizon length in order to derive a feasible optimization problem when the terminal set is $\Omega_{\bar{P}}$ or $\Omega_{\bar{P}(\nu)}$. With the first MPC choice, the MPC has not been able to solve the problem, even considering a prediction horizon up to a prediction window of $N=500$ steps, which would anyways yield a problem without a practical meaning, due to the huge dimension of the optimization problem. On the other hand, the MPC controller with $\Omega_{\bar{P}(\nu)}$ provides a solution when $N \geq 173$. This is mainly due to the difference on the size of the terminal sets $\Omega$. In addition to this, it has been showed in Fig. 2 that the size of $\Omega_{\bar{P}(\nu)}$ in the $\psi_{\text {rel }}$ dimension is very restrictive. That means that the domain of attraction for this state is relatively low, constraining the initial conditions for this angle at the $10 \%$ of its maximal value, $0.5[\mathrm{rad}]$. This means that this state will be the most sensitive from the prediction horizon point of view.

\section{B. Analysis of the IBC feasibility}

In this case the control technique is not affected by a prediction horizon but by the size and complexity of the positive invariant sets used in the formulation. This controller ensures feasibility to those points that belong to the original CIS, $\mathcal{C}_{N}$ or the scaled one $\mathcal{C}^{\prime}{ }_{N}$, (IV-B). In order to check the closed loop behavior, we have performed several simulations for a grid of initial conditions, varying from $[0,0,0,0,0]^{T}$ to the maximum values inside a $\mathbb{R}^{5}$ box. Four different IBC controllers have been tested. In Table I it can be seen that the use of a bigger MOAS, $\Omega_{\bar{P}(\nu)}$ and the scaling technique for $\mathcal{C}_{N}$ provides an improved feasibility.

TABLE I: Interpolation based control tests

\begin{tabular}{|c||c||c||c|}
\hline MOAS & $\mathcal{C}_{N}$ & $N^{\circ}$ feasible $x_{0}$ & $N^{\circ}$ unfeasible $x_{0}$ \\
\hline \multirow{2}{*}{$\Omega_{\bar{P}}$} & Fixed & $50.76 \%$ & $49.24 \%$ \\
& Scaled & $57.36 \%$ & $42.63 \%$ \\
\hline \multirow{2}{*}{$\Omega_{P(\nu)}$} & Fixed & $80.24 \%$ & $19.76 \%$ \\
& Scaled & $85.23 \%$ & $14.77 \%$ \\
\hline
\end{tabular}

\section{Numerical simulation}

In order to allow a detailed analysis, a simulation case is presented together with the time-signals. The initial conditions have been fixed to $x_{0}=[0.025,0.02,0.15,0.25,0]^{T}$, which represent a common situation in which the system would be activated. For the MPC, the prediction horizon has been fixed according to the time to collision, that is often fixed at $2[s]$. This corresponds to $N=200$ (II-B). Finally, just the acceleration phase is shown in the simulation. The first MPC (using $\bar{P}$ for the terminal cost formulation and $\Omega_{\bar{P}}$ for the terminal set constraint) has a domain of attraction which makes the MPC policy unfeasible for this case study, being unable to solve the problem for such initial conditions, so it has been discarded from the test. At the same time, the IBC controller that use a fixed $\mathcal{C}_{N}$ is not applicable: the initial point is out of the $\mathcal{C}_{N}$, so the strategies that use a fixed $\mathcal{C}_{N}$ are not applicable. In Figures 3, 4 and 5, the time-trajectory for the MPC controller using $\bar{P}(\nu)$ for the terminal cost formulation and $\Omega_{\bar{P}(\nu)}$ for the terminal set constraint and the IBC using the scaling technique are shown. In Figure 6 , the IBC interpolation factor is shown. It is interesting to note that $\eta_{k}=0$ after 2,5 [s], implying that from that time instant, the state of the closed-loop system is in the invariant set $\Omega$. Its monotonic decrease and the positivity confirms the Lyapunov interpretation given in IV-B.
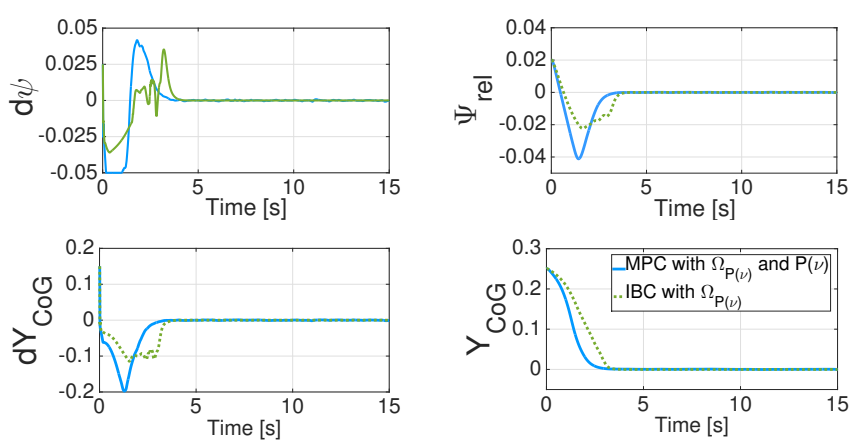

Fig. 3: States trajectories

\section{CONCLUDING REMARKS AND FUTURE WORK}

Two approaches for the design of auto-steering target tracking control have been considered, when the system dynamics are described by a parameter-varying model where the parameter, vehicle's speed, is bounded and measured. 

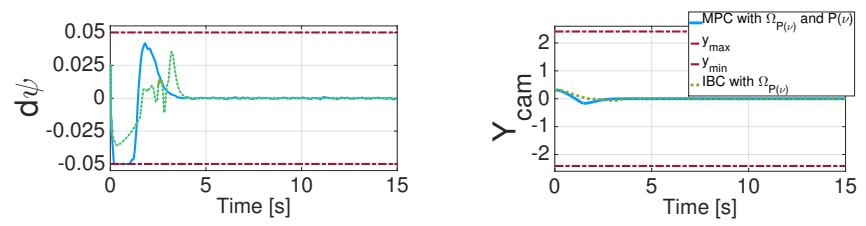

Fig. 4: Outputs trajectory
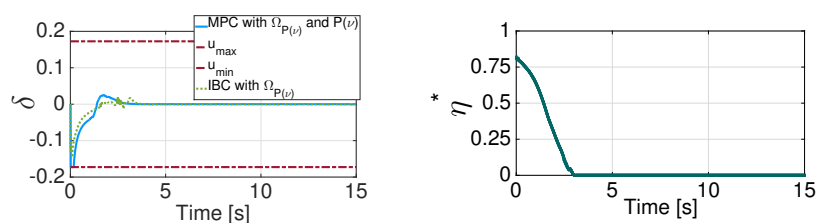

Fig. 5: Input trajectory

Fig. 6: IBC interpolation factor

[3] O. Sename, P. Gaspar, and J. Bokor, Robust control and linear parameter varying approaches: application to vehicle dynamics. Springer, 2013, vol. 437.

[4] S. Fergani, L. Menhour, O. Sename, L. Dugard, and B. Novel, "Study and comparison of non linear and LPV control approaches for vehicle stability control," in Control \& Automation (MED), 2013 21st Mediterranean Conference on. IEEE, 2013, pp. 303-310.

[5] J. Bokor and G. Balas, "Linear parameter varying systems: A geometric theory and applications," in 16th IFAC World Congress, Prague, vol. 1, 2005, pp. 1-11.

[6] A. Carvalho, S. Lefèvre, G. Schildbach, J. Kong, and F. Borrelli, "Automated driving: The role of forecasts and uncertainty-a control perspective," European Journal of Control, 2015.

[7] H. Pacejka, Tire and vehicle dynamics. Elsevier, 2005.

[8] S. Di Cairano, H. E. Tseng, D. Bernardini, and A. Bemporad, "Vehicle yaw stability control by coordinated active front steering and differential braking in the tire sideslip angles domain," Control Systems Technology, IEEE Transactions on, vol. 21, no. 4, pp. 12361248, 2013.

[9] R. Rajamani, Vehicle dynamics and control. Springer, 2006, ch. 2 Lateral Vehicle Dynamics.

On one hand the MPC has been tested, starting from a recognized technique with a mature theoretical background. The online resolution of an optimization problem in the available embedded control units for the ADAS becomes nowadays feasible due to the computational power available.

As an alternative optimization-based control, the IBC proposes a computational complexity on a par with the one step horizon MPC controller, while keeping the constraints handling feature. This makes this controller an interesting candidate for applications where the computation power available is not high. The main problem of this kind of controller is the complexity of the positive controlled invariant set, $\mathcal{C}_{N}$. Although the computation of this set is made offline and does not affect the online solver, this set increases tremendously its complexity each step we take backwards when computing it the backward reachability construction (III-B.2) thus, its size is relatively small comparing to the admissible state space set $X$. This drawback has been improved by a scaling technique that increases the domain of attraction of the interpolation based controller. However, this type of controller would not be advisable for a system with such complex positive invariant sets.

From the application point of view, the system studied in the present work has been simplified in order to provide an scenario where the attention is focused in the variation of the speed. There are more features which will be included in future controllers. These include actuator dynamics and additive disturbances, like wind forces acting on the vehicle or the curvature of the road.

\section{ACKNOWLEDGMENT}

The work leading to these results has received funding from the People Programme (Marie Curie Actions) of the European Unions Seventh Framework Programme (FP7/20072013) under REA grant agreement no 607957 (TEMPO).

\section{REFERENCES}

[1] N. M. Enache, "Assistance préventive à la sortie de voie," Ph.D. dissertation, Université d'Evry-Val d'Essonne, 2008.

[2] M. Doumiati, O. Sename, L. Dugard, J.-J. Martinez-Molina, P. Gaspar, and Z. Szabo, "Integrated vehicle dynamics control via coordination of active front steering and rear braking," European Journal of Control, vol. 19, no. 2, pp. 121-143, 2013.

[10] M. V. Kothare, V. Balakrishnan, and M. Morari, "Robust constrained model predictive control using linear matrix inequalities," Automatica, vol. 32 , no. 10 , pp. 1361-1379, 1996

[11] A. Bemporad and M. Morari, "Robust model predictive control: A survey," in Robustness in identification and control. Springer, 1999, pp. 207-226.

[12] J. B. Rawlings and D. Mayne, "Model predictive control," in The 2008 Spring National Meeting, 2008.

[13] G. Pannocchia and J. B. Rawlings, "The velocity algorithm LQR: a survey," 2001

[14] A. I. Chiriac, S. Olaru, and P. Rodriguez-Ayerbe, "A model predictive control approach for the pantograph-catenary positioning system,' Advances in Knowledge-Based and Intelligent Information and Engineering Systems, pp. 636-645, 2012.

[15] D. Q. Mayne, J. B. Rawlings, C. V. Rao, and P. O. Scokaert, "Constrained model predictive control: Stability and optimality," $\mathrm{Au}$ tomatica, vol. 36, no. 6, pp. 789-814, 2000.

[16] J. Daafouz and J. Bernussou, "Parameter dependent Lyapunov functions for discrete time systems with time varying parametric uncertainties," Systems \& control letters, vol. 43, no. 5, pp. 355-359, 2001.

[17] S. Di Cairano, "Model adjustable predictive control with stability guarantees," in American Control Conference (ACC), 2015. IEEE, 2015, pp. 226-231.

[18] W.-J. Mao, "Robust stabilization of uncertain time-varying discrete systems and comments on an improved approach for constrained robust model predictive control," Automatica, vol. 39, no. 6, pp. 11091112,2003

[19] F. Blanchini, "Survey paper: Set invariance in control," Automatica (Journal of IFAC), vol. 35, no. 11, pp. 1747-1767, 1999.

[20] E. G. Gilbert and K. T. Tan, "Linear systems with state and control constraints: The theory and application of maximal output admissible sets," Automatic Control, IEEE Transactions on, vol. 36, no. 9, pp. 1008-1020, 1991.

[21] F. Borrelli, A. Bemporad, and M. Morari, Predictive Control for linear and hybrid systems, 2015.

[22] F. Blanchini and S. Miani, Set-theoretic methods in control. Springer, 2008.

[23] "Reachability analysis and control synthesis for uncertain linear systems in MPT, author=Kvasnica, Michal and Takács, Bálint and Holaza, Juraj and Ingole, Deepak, journal=IFAC-PapersOnLine, volume $=48$, number $=14$, pages $=302-307$, year $=2015$, publisher=Elsevier.'

[24] F. Blanchini and S. Miani, Set-theoretic methods in control. Springer Science \& Business Media, 2007.

[25] H.-N. Nguyen, P.-O. Gutman, S. Olaru, and M. Hovd, "Implicit improved vertex control for uncertain, time-varying linear discretetime systems with state and control constraints," Automatica, vol. 49, no. 9, pp. 2754-2759, 2013.

[26] H.-N. Nguyen, S. Olaru, and P.-O. Gutman, "Control synthesis for switched systems with control and state constraints," in Control \& Automation (MED), 2013 21st Mediterranean Conference on. IEEE, 2013, pp. 225-230. 